\title{
Characteristics Associated with Treatment Response and Satisfaction in Women Undergoing OnabotulinumtoxinA and Sacral Neuromodulation for Refractory Urgency Urinary Incontinence
}

Holly E. Richter, ${ }^{*}$, † C. L. Amundsen, S. W. Erickson, J. E. Jelovsek, Y. Komesu, C. Chermansky, $\neq$ H. S. Harvie, M. Albo, D. Myers, W. T. Gregory and

D. Wallace for the NICHD Pelvic Floor Disorders Network

From the University of Alabama at Birmingham (HER), Birmingham, Alabama, Duke University (CLA), Durham and RTI EQ1 International (SWE, DW), Research Triangle Park, North Carolina, Cleveland Clinic (JEJ), Cleveland, Ohio, University of New Mexico (YK), Albuquerque, New Mexico, Magee-Womens Research Institute, University of Pittsburgh (CC), Pittsburgh and University of Pennsylvania (HSH), Philadelphia, Pennsylvania, University of California-San Diego (MA), San Diego, California, Brown University (DM), Providence, Rhode Island, and Oregon Health and Science University (WTG), Portland, Oregon

Purpose: We sought to identify clinical and demographic characteristics associated with treatment response and satisfaction in women undergoing onabotulinumtoxinA and sacral neuromodulation therapies.

Materials and Methods: We analyzed data from the ROSETTA (Refractory Overactive Bladder: Sacral NEuromodulation versus BoTulinum Toxin Assessment) trial. Baseline participant characteristics and clinical variables were associated with 2 definitions of treatment response, including 1) a reduction in mean daily urgency incontinence episodes during 6 months and 2) a 50\% or greater decrease in urgency incontinence episodes across 6 months. The OAB-S (Overactive Bladder-Satisfaction) questionnaire was used to assess satisfaction. Results: A greater reduction in mean daily urgency incontinence episodes was associated with higher HUI-3 (Health Utility Index-3) scores in the onabotulinumtoxinA group and higher baseline incontinence episodes (each $p<0.001$ ) in the 2 groups. Increased age was associated with a lesser decrease in incontinence episodes in the 2 groups $(\mathrm{p}<0.001)$. Increasing body mass index (adjusted OR $0.82 / 5$ points, $95 \%$ CI $0.70-0.96$ ) was associated with reduced achievement of a $50 \%$ or greater decrease in incontinence episodes after each treatment. Greater age (adjusted OR 0.44/10 years, 95\% CI 0.30-0.65) and a higher functional comorbidity index (adjusted OR $0.84 / 1$ point, 95\% CI 0.71-0.99) were associated with reduced achievement of a $50 \%$ or greater decrease in urgency incontinence episodes in the onabotulinumtoxinA group only $(\mathrm{p}<0.001$ and 0.041 ,

Accepted for publication April 27, 2017

No direct or indirect commercial incentive associated with publishing this article.

The corresponding author certifies that, when applicable, a statement(s) has been included in the manuscript documenting institutional review board, ethics committee or ethical review board study approval; principles of Helsinki Declaration were followed in lieu of formal ethics committee approval; institutional animal care and use committee approval; all human subjects provided written informed consent with guarantees of confidentiality; IRB approved protocol number; animal approved project number.

Supported by the Eunice Kennedy Shriver National Institute of Child Health and Human Development and the National Institutes of Health Office of Research on Women's Health; ClinicalTrials.gov number, NCT01502956.

* Correspondence: 1700 6th Av. South, Suite 10382, Birmingham, Alabama 35233 (telephone: 205-934-1704; FAX: 205-975-8893; e-mail: hrichter@uabmc.edu).

† Financial interest and/or other relationship with Society of Gynecologic Surgeons, UpToDate, Astellas, Ferring, Statking, Kimberly Clark, GlaxoSmithKline, Uromedical, IDEO, Pelvalon, Pfizer/University of California-San Francisco and Warner Chilcott.

¥ Financial interest and/or other relationship with Allergan and Medtronics. http://dx.doi.org/10.1016/j.juro.2017.04.103 Vol. 198, 1-7, October 2017 Printed in U.S.A.

\section{Abbreviations and Acronyms \\ $\mathrm{BMI}=$ body mass index \\ $\mathrm{FCl}=$ Functional Comorbidity Index \\ $\mathrm{HROoL}=$ health related $\mathrm{OoL}$ \\ HUI-3 = Health Utility Index-3 \\ $\mathrm{OABq}=$ Overactive Bladder \\ Questionnaire \\ $0 A B q-S F=0 A B q-S h o r t$ Form \\ $\mathrm{OoL}=$ quality of life \\ ROSETTA = Refractory Overactive Bladder: Sacral NEuromodulation versus BoTulinum Toxin \\ Assessment \\ SNM = sacral neuromodulation \\ UIE = urinary incontinence \\ episode \\ $\mathrm{UUI}=$ urgency urinary \\ incontinence \\ UUIE $=$ UUI episode}


respectively). In the onabotulinumtoxinA group increased satisfaction was noted with higher HUI score $(\mathrm{p}=0.002)$ but there was less satisfaction with higher age $(\mathrm{p}=0.001)$.

Conclusions: Older women with multiple comorbidities, and decreased functional and health related quality of life had decreased treatment response and satisfaction with onabotulinumtoxinA compared to sacral neuromodulation for refractory urgency incontinence.

Key Words: urinary bladder; urinary incontinence, urge; onabotulinumtoxinA; electric stimulation; quality of life

WOMEN with UUI refractory to primary treatment approaches, including medications and behavioral therapy, may be offered third line treatment options such as posterior tibial nerve stimulation, SNM or onabotulinumtoxinA. Understanding patient clinical and demographic characteristics potentially associated with the third line treatment response, especially in the setting of more invasive treatment approaches such as SNM and onabotulinumtoxinA, is important and may help optimize treatment success.

The ROSETTA trial was an open label, randomized trial in women with idiopathic refractory UUI who were randomized to onabotulinumtoxinA or SNM. ${ }^{1}$ The objective of this planned secondary analysis was to identify baseline clinical and demographic factors associated with treatment response and satisfaction in women participating in this multicenter randomized trial.

\section{MATERIALS AND METHODS}

The design and primary results of the ROSETTA trial have been previously published. ${ }^{1,2}$ The institutional review board at each clinical site and coordinating center approved the protocol and participants provided written informed consent. Major inclusion criteria for women participating in the ROSETTA trial included a minimum of 6 UUIEs on a baseline 3-day bladder diary, not taking or stopping UUI medications at least 3 weeks prior to the baseline evaluation and urodynamic assessment within 18 months before randomization. Standardized demographic, clinical data and key procedural elements were collected at baseline.

Briefly, participants randomized to SNM underwent stage 1 lead placement by experienced surgeons in the operating suite. During the 7 to 14-day testing phase participants with $50 \%$ or greater improvement in mean UUIE on a 3-day bladder diary were categorized as clinical responders and were eligible for placement of the permanent implantable pulse generator. Those without this improvement had the lead removed. Participants randomized to onabotulinumtoxinA received a single cystoscopic intradetrusor injection of $200 \mathrm{U}$ performed in clinic. Women with a $50 \%$ or greater reduction in UUIE on a bladder diary 1 month after injection were defined as onabotulinumtoxinA clinical responders.

Treatment response outcomes for this planned secondary analysis included 1) a reduction in mean daily
UUIEs averaged across 6 months, 2) a 50\% or greater decrease in UUIE on each completed diary across 6 months as recorded in monthly 3 -day bladder diaries and 3) treatment satisfaction on OAB-SATq (Overactive Bladder Satisfaction with Treatment Questionnaire). ${ }^{2}$

All participants with 1 to 6 post-baseline diaries were included in the mean daily UUIE analyses while only participants with at least 4 completed diaries were included in the $50 \%$ or greater decrease analyses. The potential variables thought to be associated with treatment response were sociodemographic characteristics, including age in years and race/ethnicity; medical history and functional status, including BMI as a continuous variable, smoking status, menopausal status, and the Timed Up and Go test; characteristics of urinary incontinence, including UUIE, Sandvik score and UDI-SF (Urogenital Distress Inventory-Short Form); symptom impact and incontinence related quality of life on OABq$\mathrm{SF}$ ) and IIQ-SF (Incontinence Impact QuestionnaireShort Form), and health related quality of life on HUI-3; and urodynamic variables and medical comorbidities, including history of recurrent urinary tract infections and FCI. ${ }^{2,3}$

To identify variables associated with a reduction in mean daily UUIEs during 1 to 6 months after treatment as a continuous outcome we fit linear mixed models for each potential predictor, controlling for treatment group, age group (less than 65 and 65 years or greater), site, month after treatment and interaction of treatment group with month. Person-month was used as the unit of analysis. Participants were treated as a random effect to account for the within subject correlation with time.

To evaluate whether the impact of the potential predictors differed by treatment group the models were fit with a treatment by predictor interaction term. To identify baseline variables associated with a $50 \%$ or greater reduction in UUIEs as a dichotomous outcome logistic regression models were fit for each potential predictor with all models controlling for treatment group and age group as factors included in the original randomization. An analogous linear model was used to examine the effect of predictors on treatment satisfaction using OAB-SATq at 6 months.

Initially each baseline variable was modeled individually to assess associations with each outcome. Parameter estimates, ORs and $95 \%$ CIs were generated by treatment group based on treatment stratified analyses. Variables in which the main effect or the interaction term was at $\mathrm{p}<0.10$ using the Wald test were included as candidate terms in a combined multivariable model. That model 
then underwent backward variable selection so that each term in the final multivariable model was at $\mathrm{p}<0.10$. Results were not adjusted for multiple comparisons and all $\mathrm{p}$ values should be interpreted accordingly. Analyses were performed with $\mathrm{SAS} \circledast$, version 9.4.

\section{RESULTS}

A total of 190 participants randomized to onabotulinumtoxinA and 174 randomized to SNM were included in this secondary analysis. Baseline characteristics were similar in the treatment groups (supplementary table 1, http://jurology.com/). Overall mean $\pm \mathrm{SD}$ age was $6 \overline{3.0 \pm 11.6 \text { years and }}$ mean body mass index was $32.2 \pm 8.2 \mathrm{~kg} / \mathrm{m}^{2}$. Participants reported a mean of $5.30 \pm 2.67$ UUIEs per day and $96 \%$ reported that UUI symptoms were moderate or greater on the Sandvik questionnaire. Supplementary table 1 (http://jurology.com/) lists other characteristics.

The reduction in mean daily UUIEs during 6 months was not associated with most baseline covariates on univariable analysis. However, increasing age and higher (better) baseline OABqSF QoL score were associated with a lesser decrease in mean daily UUIEs during 6 months (supplementary table 2, http://jurology.com/). Hispanic/Latina ethnicity, detrusor overactivity on cystometrogram, a greater number of daily UUIEs and total UIEs per day, higher (worse) OABq-SF symptom bother scores and higher (better) HUI-3 scores were associated with a greater reduction in mean daily UUIEs.

A greater effect was noted in the onabotulinumtoxinA group for UUIEs and total UIEs. Multivariable analyses revealed that age and greater baseline UUIE frequency in the onabotulinumtoxinA and SNM groups were associated with a TT1 UUIE reduction (tables 1 to 3 ). For age there was an average decrease in reduction of about 0.3 UUIEs per day for each added decade of age. For greater baseline UUIE frequency there was an increased reduction of 0.6 UUIEs per day for each unit increase in baseline UUIEs, ie an increased reduction of approximately $60 \%$ of the incremental level. Increasing age was associated with a lower treatment response while higher frequency of baseline UUIEs was associated with an increased treatment response. Higher HUI-3 score was also associated with an improved treatment response with some evidence of a greater reduction in UUIEs in the onabotulinumtoxinA group.

Similarly univariable analysis of the achievement of a $50 \%$ or greater reduction in UUIEs revealed that increased age, higher BMI, higher (worse) FFCI score, higher (worse) baseline IIQ-SF score and higher (worse) Sandvik score were associated with decreased odds (supplementary table 3, http://jurology.com/). Greater volume at maximum cystometric capacity and higher (better) HUI-3 score were associated with increased odds of achieving a $50 \%$ or greater reduction in UUIEs. A greater effect (lower OR) was noted in the onabotulinumtoxinA group for FCI.

On multivariable analysis increasing age and higher BMI were associated with decreased odds of achieving a treatment response of a $50 \%$ or greater reduction in UUIEs with the effect of age greater in the onabotulinumtoxinA group. Higher FCI score was associated with decreased odds of achieving a $50 \%$ or greater reduction in UUIEs only in the onabotulinumtoxinA group.

Univariable analysis revealed that for each treatment greater age, UUIEs per day, total UIEs per day and Sandvik score were associated with decreased treatment satisfaction, as was Latina ethnicity (supplementary table 4, http://jurology. com/). African American race was associated with episodes in posttreatment months 1 to 6

\begin{tabular}{|c|c|c|c|c|}
\hline & \multicolumn{2}{|c|}{ Coefficient (95\% Cl) } & \multicolumn{2}{|c|}{$p$ Value } \\
\hline & OnabotulinumtoxinA & $\begin{array}{c}\text { Sacral } \\
\text { Neuromodulation }\end{array}$ & $\begin{array}{l}\text { Main Predictor } \\
\text { Effect }\end{array}$ & Treatment $\times$ Predictor \\
\hline \multicolumn{5}{|c|}{ Mean UUIEs/day reduction:* } \\
\hline No. pts & 190 & 174 & - & - \\
\hline HUI-3/0.30 points & $(0.23-0.77)$ & $0.09(-0.21-0.40)$ & 0.004 & 0.051 \\
\hline \multicolumn{5}{|c|}{ OABq treatment satisfaction: } \\
\hline No. pts & 150 & 129 & - & - \\
\hline Age/decade & $-6.53(-10.39--2.68)$ & $-0.69(-4.86-3.47)$ & 0.015 & 0.039 \\
\hline HUI-3/0.30 points & $(2.48-11.19)$ & $0.41(-4.79-5.60)$ & 0.039 & 0.059 \\
\hline
\end{tabular}

* Final model included HUI-3 per 0.1 point, age in decades to generate meaningful effect estimates (age included in model as continuous variable in years) and UUIEs per day.

EQ2 †HUI-3 baseline standard deviation in our study population and HUI-3 effect in onabotulinumtoxinA and sacral neuromodulation groups $p<0.001$ and $p=0.545$, respectively.

$\ddagger$ Final model included HUI-3 per 0.1 point (baseline standard deviation in our study population), age in decades, UUIEs and stress UIEs per day, and race, and age and HUI-3 effects of $p=0.001$ and 0.002 in onabotulinumtoxinA group, and $p=0.744$ and 0.878 , respectively, in sacral modulation group. 
Table 2. Multivariable associations between participant characteristics and reduction in mean daily urgency urinary incontinence episodes in posttreatment months 1 to 6

\begin{tabular}{|c|c|c|}
\hline & $\begin{array}{c}\text { OnabotulinumtoxinA + Sacral } \\
\text { Neuromodulation } \\
\text { Coefficient }(95 \% \mathrm{CI})\end{array}$ & $\begin{array}{l}\text { Main Predictor } \\
\text { Effect } p \text { Value }\end{array}$ \\
\hline \multicolumn{3}{|c|}{ Mean UUIEs/day reduction* } \\
\hline $\begin{array}{l}\text { No. pts } \\
\text { Age/decade } \\
\text { No. UUIEs/day }\end{array}$ & $\begin{array}{l}364 \\
-0.32(-0.49--0.15) \\
\quad 0.62 \quad(0.54-0.70)\end{array}$ & $\begin{array}{l}- \\
<0.001 \\
<0.001\end{array}$ \\
\hline \multicolumn{3}{|c|}{$O A B q$ treatment satisfactiont } \\
\hline $\begin{array}{l}\text { No. pts } \\
\text { Race: }\end{array}$ & 279 & - \\
\hline $\begin{array}{l}\text { African American } \\
\text { Other } \\
\text { Caucasian } \\
\text { No. UIEs/day: }\end{array}$ & $\begin{array}{rr}14.62 & (3.41-25.83) \\
-6.91 & (-19.87-6.05) \\
& \text { Referent }\end{array}$ & 0.017 \\
\hline $\begin{array}{l}\text { Urgency } \\
\text { Stress }\end{array}$ & $\begin{array}{l}-1.89(-3.25--0.54) \\
-4.65(-8.81--0.49)\end{array}$ & $\begin{array}{l}0.006 \\
0.029\end{array}$ \\
\hline
\end{tabular}

* Final model included HUI-3 score per 0.1 points, age in decades to generate meaningful effect estimates (age included in model as continuous variable in years) and urgency urinary incontinence episodes per day.

†Final model included HUl-3 score per 0.1 points, age in decades to generate meaningful effect estimates (age included in model as continuous variable in years), UUIEs per day, stress UIEs per day and race.

increased satisfaction relative to Caucasian race. Increased HUI score was associated with increased satisfaction in the onabotulinumtoxinA group.

Multivariable analysis revealed that in each treatment group African American race was associated with increased satisfaction, and increased UUIEs per day and stress UIEs per day with decreased satisfaction. Increased age was associated with decreased satisfaction, a higher HUI score and

Table 3. Multivariable associations of participant characteristics with $50 \%$ or greater UUIE reduction during 6 months

Adjusted OR $(95 \% \mathrm{CI})$

\begin{tabular}{ll}
\hline No. pts: & \\
OnabotulinumtoxinA & 178 \\
Sacral neuromodulation & 166 \\
Age (decades): $t$ & \\
OnabotulinumtoxinA & $0.44(0.30-0.65)$ \\
Sacral neuromodulation & $0.78(0.57-1.08)$ \\
Main predictor effect $p$ value & $<0.001$ \\
Treatment $\times$ predictor $p$ value & 0.016 \\
OnabotulinumtoxinA + sacral neuromodulation: & \\
BMl kg/m $/ 5$ points & $0.82(0.70-0.96)$ \\
Main predictor effect $p$ value & 0.013 \\
IIQ/10 points & $0.91(0.82-1.00)$ \\
Main predictor effect $p$ value & 0.062 \\
Sandvik score & $0.92(0.84-1.01)$ \\
Main predictor effect $p$ value & 0.068
\end{tabular}

Final model included age in decades to generate meaningful effect estimates (age included in model as continuous variable in years), $\mathrm{FCl}$ per per point, body mass index in $\mathrm{kg} / \mathrm{m}^{2}$ per 5 points, $I 10$ per 10 points and Sandvik score.

* Patients completed at least 4 bladder diaries.

$\dagger$ In onabotulinumtoxinA group age effect $p<0.001$ and $\mathrm{FCl}$ effect $p=0.041$, and in sacral neuromodulation group $p=0.137$ and 0.385 , respectively. greater satisfaction with onabotulinumtoxinA treatment.

\section{DISCUSSION}

In this planned secondary analysis of women with refractory UUI randomized to onabotulinumtoxinA or SNM increasing age was associated with a lesser mean reduction (less efficacy) while higher baseline HUI-3 score and UUIEs were associated with an increased mean reduction (greater efficacy) in UUIEs per day with the HUI-3 effect greater in the onabotulinumtoxinA group. Similarly regarding the outcome of a $50 \%$ or greater reduction in UUIEs increasing age and higher BMI were associated with decreased odds of a treatment response in all participants. A higher FCI score conferred decreased odds only in the onabotulinumtoxinA group.

These 2 specific treatment outcomes were studied since they are commonly reported in the onabotulinumtoxinA and SNM literature. Overall treatment satisfaction with these third line therapies was associated with African American race and decreased satisfaction was associated with increased UUIEs and stress UIEs. Similar to treatment responsiveness increased age was associated with less satisfaction in the onabotulinumtoxinA group. Better health related QOL was associated with greater satisfaction with onabotulinumtoxinA therapy.

Increasing age was independently associated with a poorer treatment response to each treatment and for each UUIE treatment response definition used in this study. The age association suggests that lower urinary tract changes which develop with aging, including decreased urethral closure pressure and detrusor contractility, and increased detrusor overactivity, may predispose older women to a UUI phenotype that may be more refractory to treatment. ${ }^{4-6}$

To provide perspective on average a 55 -year-old woman would have an increased mean reduction of 0.64 UUIEs per day compared to a 65-year-old woman. In the onabotulinumtoxinA group a 55year-old woman would have about twice the odds of achieving a $50 \%$ or greater reduction in UUIEs compared to a 65-year-old woman. The effect of age in previous reports of SNM treatments has varied. Prior SNM studies described older women gaining benefit from SNM but showed higher continence rates and a greater UUIE reduction in the younger group. ${ }^{7,8}$ In contrast, Peters et al prospectively evaluated 328 patients, of whom $83 \%$ were women, and found that SNM success was not age dependent. ${ }^{9}$ Including an urgency only population and pudendal neuromodulation may have affected the latter results. 
Less information exists regarding the effect of age on onabotulinumtoxinA treatment response and satisfaction. One group reported results in 27 patients with refractory UUI after comparing outcomes in younger and older patients (mean age $55 \pm$ 15 and $68 \pm 13$ years, respectively). ${ }^{10}$ Although younger age predicted a $50 \%$ or greater UUIE reduction on univariate analysis, age was not significant on multivariable analysis. In the current study we found a differential treatment effect with respect to age and the $50 \%$ or greater UUIE reduction treatment outcome, which to our knowledge has not been reported previously. With increasing age women undergoing onabotulinumtoxinA treatment had a decreased treatment response compared to those undergoing SNM. This differential effect of treatment type was not noted for the outcome of reductions in mean daily incontinence episodes during 6 months with increasing age mitigating the response to each treatment.

In the current study we found that increased baseline BMI decreased the odds of achieving a 50\% or greater UUIE reduction. For example, the model estimated that a woman with a BMI of $25 \mathrm{~kg} / \mathrm{m}^{2}$ would have about $50 \%$ greater odds of achieving a $50 \%$ or greater reduction in UUIEs than a woman with a BMI of $35 \mathrm{~kg} / \mathrm{m}^{2}$. This is consistent with prior epidemiological literature in which increasing BMI was associated with UUI severity. ${ }^{11,12}$ Determining the association of BMI for predicting treatment success is important because it is a modifiable risk factor. Each 5-point increase in baseline BMI decreased the odds of attaining a $50 \%$ or greater UUIE reduction by approximately $20 \%$. Alternatively a woman with a BMI of $25 \mathrm{~kg} / \mathrm{m}^{2}$ would have about $50 \%$ greater odds of achieving a $50 \%$ or greater reduction in UUIEs than a woman with a BMI of $35 \mathrm{~kg} / \mathrm{m}^{2}$, assuming that all other covariates were the same. Subak et al found that weight loss after bariatric surgery was associated with substantially decreased urinary incontinence during 3 years. ${ }^{13}$ Further research is needed of the role of weight loss in UUI refractory to treatment.

Other characteristics associated with treatment response included the baseline number of UUIEs per day and HUI-3 in the overall reduction in mean daily UUIEs. Women with higher baseline UUIEs per day experienced a greater mean decrease in UUIEs per day. This finding reinforces previous results, including those in a randomized trial of anticholinergic therapy vs onabotulinumtoxin $\mathrm{A}^{14}$ and another describing the results of SNM testing. ${ }^{15}$

HUI-3 is an instrument used to measure general health status and HRQoL which is scored on a scale of 0.00 - death to 1.00 - perfect health. ${ }^{2}$ HUI-3 has been noted to provide valid measurements for utility scores in women with stress, urgency and mixed urinary incontinence. ${ }^{16}$ In our study HUI-3 demonstrated responsiveness to a change in the treatment of refractory UUI in the onabotulinumtoxinA and SNM groups. Higher baseline HUI3 scores were associated with a better treatment response. For example, a woman with a baseline HUI-3 score of 0.7 would experience an average reduction of 0.5 episodes per day more compared to a woman with a baseline score of 0.4 . This represents a difference of 0.3 in HUI- 3 score, which is approximately the baseline standard deviation in our study population and is 10 times the reported minimally important difference of $0.03 .^{17}$

Women with higher health related quality of life had a greater improvement in the mean daily reduction in UUIEs during 6 months in each treatment group with greater decrease in the onabotulinumtoxinA group. This may reflect a differential impact of incontinence on general health status and HRQoL, and a greater chance of treatment benefit. The trend toward a greater beneficial effect of a higher HUI-3 score on the success of onabotulinumtoxinA is unclear. Further research is again warranted to understand the particular interaction between botulinum toxin and health status.

Finally, with respect to FCI and its association with a $50 \%$ or greater reduction in UUIEs in the onabotulinumtoxinA group, women with more medical comorbidities have a greater likelihood of increased incontinence severity. ${ }^{18,19}$ In the current study a woman with a baseline FCI score of 1 would have approximately $70 \%$ greater odds of achieving a $50 \%$ or greater reduction in UUIEs than a woman with a baseline score of 4 . Assessment of baseline physical function and HRQoL status as measured by FCI is important since it may be used to control for baseline comorbidity, provide a perspective on potential return to functional levels after treatment and serve as a predictor of outcome. ${ }^{20}$ Incontinence treatment may be more difficult in women with more morbidities due to the potential cumulative negative impact on daily functioning and bladder control. This was previously noted in 105 men and women in whom decreased SNM cure rates were associated with having 3 or more chronic medical conditions regardless of age. ${ }^{8}$ It is unclear why we found a differential treatment effect for onabotulinumtoxinA. As with HUI-3, further evaluation is needed to better understand this specific finding.

Our planned secondary analysis had several strengths. It allowed robust assessment of a large number of women who met standardized criteria for refractory UUI with clearly defined preoperative clinical and demographic variables, and validated outcome measures, treatment outcomes and satisfaction. Study limitations include a followup limited 
to 6 months and the inclusion of only women participants.

\section{CONCLUSIONS}

Older women with multiple comorbidities, and decreased functional and health related QoL had reduced treatment response and satisfaction with onabotulinumtoxinA compared to sacral neuromodulation for refractory urgency incontinence. Increasing BMI was associated with a decreased response to each treatment. This information may help individualize treatment approaches for these more invasive third line therapies.

\section{ACKNOWLEDGMENTS}

Drs. Patricia S. Goode, Robert L. Holley, L. Keith Lloyd, Alayne D. Markland, Robert E. Varner and Tracey S. Wilson, and Julie E. Burge, Kathy S. Carter, Ryanne R. Johnson, R. Jeannine McCormick, Nancy B. Saxon, Velria B. Willis and Robin R. Willingham, Univesity of Alabama at Birmingham;
Alexandra Lynch, Ann Meers, Allegra Parrillo and Erika Spearin, Brown University; JoAnn Columbo, University of California-San Diego; Nancy Flores, Kaiser Downey; Gisselle Zazueta-Damian, Kaiser San Diego; Dr. Bradley Gill, Andrea Aaby and Ly Pung, Cleveland Clinic; Drs. Amie Kawasaki, Nazema Y. Siddiqui, Anthony G. Visco and Alison C. Weidner, and Akira Hayes, Nicole Longoria and Shantae McLean, Duke University; Julie Middendorf and Karen Taylor, University of New Mexico; Michelle Kinglee, University of Pennsylvania; Drs. Michael Bonidie, Pamela Moalli, Jonathan Shepherd and Gary Sutkin, and Christopher Chermansky, Judy Gruss and Karen Mislanovich, University of Pittsburgh; and Maura Bearden, Katrina Burson, Allyson Drew, Marie Gantz, Kendra Glass, Rae Hope, Carolyn Huitema, Amy Kendrick, Daryl Matthews, Tracy Nolen, James Pickett, Kelly Roney, Amanda Shaffer, Yan Tang, Ryan Whitworth, Kevin Wilson, Anita Woodring, RTI International, assisted with the study.

\section{REFERENCES}

1. Amundsen $\mathrm{CL}$, Richter $\mathrm{HE}$, Menefee $\mathrm{SA}$ et al: OnabotulinumtoxinA vs sacral neuromodulation on refractory urgency urinary incontinence in women: a randomized clinical trial. JAMA 2016; 316: 1366 .

2. Amundsen $\mathrm{CL}$, Richter HE, Menefee $\mathrm{S}$ et al: The Refractory Overactive Bladder: Sacral Neuromodulation vs BoTulinum Toxin Assessment: ROSETTA Trial. Contemp Clin Trials 2014; 37: 272.

3. Groll DL, To T, Bombardier $C$ et al: The development of a comorbidity index with physical function as the outcome. J Clin Epidemiol 2005; 58: 595

4. DuBeau CE, Kuchel GA, Johnson T II et al: Incontinence in the frail elderly: report from the 4th International Consultation on Incontinence. Neurourol Urodyn 2010; 29: 165

5. DuBeau CE: Therapeutic/pharmacologic approaches to urinary incontinence in older adults. Clin Pharmacol Ther 2009; 85: 98.

6. DuBeau CE: The aging lower urinary tract. J Urol 2006; 175: S11.

7. Amundsen CL and Webster GD: Sacral neuromodulation in an older, urge-incontinence population. Am J Obstet Gynecol 2002; 187: 1462.
8. Amundsen $\mathrm{C}$, Romero $\mathrm{A}$, Jamison $\mathrm{MG}$ et al: Sacral neuromodulation for intractable urge incontinence: are there factors associated with cure? Urology 2005; 66: 746.

9. Peters KM, Killinger KA, Gilleran $J$ et al: Does patient age impact outcomes of neuromodulation? Neurourol Urodyn 2013; 32: 30.

10. Cohen BL, Caruso DJ, Kanagarajah P et al: Predictors of response to intradetrusor botulinum toxin-A injections in patients with idiopathic overactive bladder. Adv Urol 2009; 328364.

11. Waetjen LE, Liao S, Johnson WO et al: Factors associated with prevalent and incident urinary incontinence in a cohort of midlife women: a longitudinal analysis of data. Study of Women's Health Across the Nation. Am J Epidemiol 2006; 165: 309

12. Minassian VA, Devore E, Hagan K et al: Severity of urinary incontinence and effect on quality of life in women by incontinence type. Obstet Gynecol 2013; 121: 1083.

13. Subak LL, King WC, Belle SH et al: Urinary incontinence before and after bariatric surgery. JAMA Intern Med 2015; 175: 1378.

14. Visco AG, Brubaker L, Richter HE et al: Anticholinergic therapy vs onabotulinumtoxinA for urgency urinary incontinence. $\mathrm{N}$ Engl $\mathrm{J}$ Med 2012; 367: 1803

15. Yazdany T, Bhatia N and Nguyen J: Determining outcomes, adverse events, and predictors of success after sacral neuromodulation for lower urinary disorders in women. Int Urogynecol $J$ 2011; 22: 1549

16. Harvie HS, Shea JA, Andy UU et al: Validity of utility measures for women with urge, stress, and mixed incontinence. Am J Obstet Gynecol 2014; 210: 85.e1.

17. Drummond M: Introducing economic and quality of life measurements into clinical studies. Ann Med 2001; 33: 344

18. Ragins Al, Shan J, Thom DH et al: Effects of urinary incontinence, comorbidity and race on quality of life outcomes in women. J Urol 2008; 179: 651

19. Melville JL, Katon W, DeLaney K et al: Urinary incontinence in women: a population based study. Arch Intern Med 2005; 165: 537

20. Orwelius L, Nordland A, Nordlund P et al: Preexisting disease: the most important factor for health related quality of life long-term after critical illness: a prospective, longitudinal, multicenter trial. Crit Care 2010; 14: R67. 


\section{EDITORIAL COMMENT}

In this preplanned secondary analysis of the ROSETTA trial ${ }^{1}$ Richter et al state that higher age and a higher comorbidity index were associated with a decreased response rate to onabotulinumtoxinA injections on multivariable analysis. This relatively new finding again raises the issue of patient selection when considering second line management of overactive bladder.

One important limitation of the analysis is that women with nonneurogenic (but maybe not idiopathic) overactive bladder were included. Indeed, nonneurogenic overactive bladder is rather a multifactorial disease with poorly understood underlying causes. Bowel disease, pelvic organ chronic dysfunction or crosstalk, asymptomatic neurological diseases, an autonomous nervous system imbalance, concomitant medications, the brain-bladder axis, urinary microbiota, psychological conditions and several other factors with unclear respective roles might explain overactive bladder and influence treatment outcomes. Refining our diagnostic approach might be the price to pay to better select patients and make a step forward in this field.

\section{Jean-Nicolas Cornu* \\ Charles Nicolle University Hospital} Rouen Cedex, France

*Financial interest and/or other relationship with Allergan, Astellas, Boston Scientific, Bouchara-Recordati, Coloplast, Cousin Biotech, Medtronic, Mundipharma, Pfizer, Pierre Fabre, SAP, Takeda, GT Urological and Ipsen. 